\title{
Spin-resolved off-normal photoemission from xenon adsorbates in comparison with free-atom photoionization *
}

\author{
B. Kessler, N. Müller, B. Schmiedeskamp, B. Vogt, and U. Heinzmann \\ Fakultät für Physik, Universität Bielefeld, D-4800 Bielefeld, Federal Republic of Germany and Fritz-Haber-Institut der MPG, \\ D-1000 Berlin 33, Germany
}

Received 27 June 1990

Circularly polarized synchrotron radiation of BESSY has been used to study the angular dependence of the spin polarization of photoelectrons emitted from $\mathrm{Xe}(\sqrt{3 x} \sqrt{3}) R 30^{\circ} \mathrm{Pd}(111)$. The spin-polarization component along the light helicity varies from +1 for normal emission to -0.5 for emission angles larger than $45^{\circ}$. The data can be fitted by use of the theory for photoionization of free xenon atoms and yield the dynamical fit parameters $A, \alpha, \beta, \xi$ for the adsorbate. Good agreement is obtained with the gas phase data.

PACS: $32.80 . \mathrm{Fb} ; 71.70 . \mathrm{Ej} ; 79.60 . \mathrm{Gs}$

\section{Introduction}

Energy-, angle, and spin-resolved photoemission studies on adsorbate systems were up to now restricted to the highly symmetrical set up of normal light incidence and normal photoelectron emission [1-3]. By use of the circularly polarized synchrotron radiation at BESSY in Berlin, the photoemission from the valence bands of rare gas adlayers on metal single crystals yielded a complete spin polarization of photoelectrons parallel or antiparallel to the photon spin. Thus a direct assignment of the symmetries of the electronic states of the adsorbates was possible [4-7]. Similar normal-emission experiments at non-ferromagnetic single crystal surfaces [8-13] allowed to characterize the symmetry of the bands involved and to perform a symmetry-resolved band mapping. The spin effects discussed here are based on the spin-orbit interaction and partly depend on the relativistic selection rules for dipole transitions with circularly polarized radiation [14-16]. In contrast to this, spin effects in photoemission from ferromagnetic solids [17] are primarily an effect of the initial states spin split due to the exchange interaction. * This article is dedicated to Professor Dr. J. Kessler, Münster
on the occasion of his 60 th birthday
Spin-resolved off-normal photoemission from solids (described in a three step model) contains the problem to distinguish between spin dependent effects in the photoelectron excitation, in the transport to the surface, and in the transmission at the surface [14-16]. This has been experimentally studied for off-normal photoemission from $\mathrm{Pt}(111)$ [18-20]. Considering these results and to disregard spin effects due to photoelectron diffraction [21] the experiments with adsorbates concerning the present paper have been performed with the centro-symmetrical medium $Z$ substrate $\operatorname{Pd}(111)$. Furthermore, the photoelectron emission was studied in the mirror plane of adsorbate and substrate. For this condition electron scattering only yields a spin-polarization component normal to the scattering plane, comparable to a single scattering process $[14-16,22]$. Additionally we used Xe as adsorbate system because for a Xe single crystal the experimental LEED results can be well fitted within a single scattering (kinematic) approximation [23]. Finally, our adsorbate system was the well ordered $\mathrm{Xe}(\sqrt{3 x} \sqrt{3}) R 30^{\circ}$ monolayer on $\operatorname{Pd}(111)$. The photoelectrons studied are thus produced at the outermost atoms within the interface solid/vacuum.

It is the purpose of this paper to report on the first experiment of spin-resolved off-normal photoemission from an adsorbate and to quantitatively compare the results within an atomlike model with existing data of free atom photoionization also obtained at BESSY [24, $25]$. This cross comparison is done, because a quantitative relativistic one step photoemission theory for adsorbates is not yet available and because at the Xe adsorbate the kinematic model is expected to be a good first approximation. The experimental study of angle-, spin, and energy-resolved photoionization of free Xe atoms enabled a quantum mechanical complete characterization, i.e. an experimental determination of all allowed matrix elements and phase-shift differences of the continuum wavefunctions. The direct comparison of the spinresolved photoionization results in the gas phase with photoemission from the adsorbate system should also answer the question, whether free-atom like phase-shift 
differences of final states and matrix element effects may also describe the photoelectron emission from a monolayer adsorbed.

\section{Experimental set up}

The measurements were carried out at the $6.5 \mathrm{~m}$ normal incidence monochromator [26] of the electron storage ring BESSY. A description of the experimental set up is given elsewhere $[1,2,10]$. The circularly polarized off-storage-ring plane synchrotron radiation hits normally the adsorbate system $\mathrm{Xe}(\sqrt{3 x} \sqrt{3}) R 30^{\circ} / \mathrm{Pd}(111)$, which was prepared at a base pressure of $4 \times 10^{-11} \mathrm{mbar}$ and a temperature of $65 \pm 5 \mathrm{~K}$ on a LHe-cooled manipulator. The adsorbate geometry was controlled by LEED. The photoelectrons ejected are angle- and energy-analysed by a rotatable electron spectrometer [27]. The overall energy resolution was better than $150 \mathrm{meV}$ at an angular resolution of $\pm 3^{\circ}$. Two electron spin polarization components $A(\Theta)$ (parallel to the light helicity) and $P_{\perp}(\Theta)$ (perpendicular to the reaction plane, given by the momenta of light and electrons at the target) as defined in the left part of Fig. 1 are simultaneously determined by Mott scattering [22]. As in practice the synchrotron radiation is slightly elliptically polarized (degree of circular polarization $\pm 93 \%$ ), one has to take into account the azimuthal orientation $\phi$ of the light ellipse. For an orientation of $\phi=45^{\circ}$ of the reaction plane with respect to the principal axes of the ellipse, for free atoms the spin polarization components depend on the light polarization by simple relations $[24,25]$. Thus we have chosen the same geometry for the adsorbate studies, too. Fig. 1 explains the symmetry behaviour of the gas-phase photoionization plane as well as the reaction plane of adsorbate photoemission (described by the left part of Fig. 1). Our results presented below are dominated by the photoexcitation process within the experimental uncertainties and not by the transmission through the surface: photoelectron intensities and polarizations indeed do not show asymmetries when changing the emission angle from $+\Theta$ to $-\Theta$ or switching the light helicity from $\sigma^{+}$to $\sigma^{-}$apart from the sign change of the spin polarization.

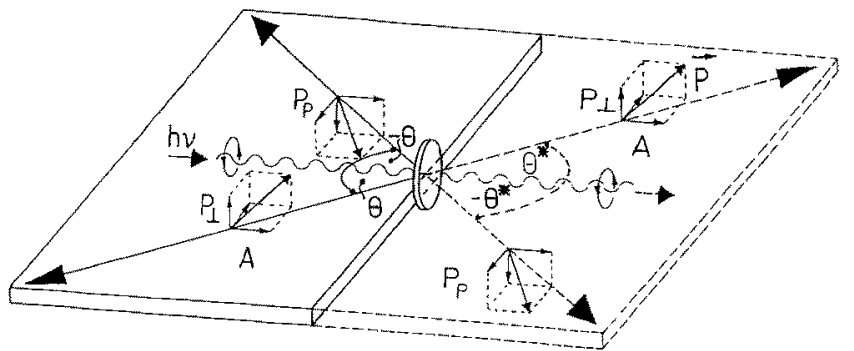

Fig. 1. Reaction plane of photoelectron emission given by the momenta of incoming circularly polarized photon and outgoing photoelectron. $A(\Theta), P_{p}(\Theta)$ and $P_{\perp}(\Theta)$ are the three components of the spinpolarization vector $P$ of photoelectrons. The left part denotes the reaction plane of adsorbate-photoemission with the target as shown. The full reaction plane is valid for photoionization of an isolated atom in the center with $\Theta^{*}$ as emission angle

\section{Experimental results of adsorbate photoemission in cross comparison with gas phase data}

Gas-phase photoionization of $\mathrm{Xe} 5 p^{6}\left({ }^{1} S_{0}\right)$ leads to $\mathrm{Xe}^{+} 5 p^{5}\left({ }^{2} P_{3 / 2,1 / 2}\right)$ called the $p_{3 / 2}$ and $p_{1 / 2}$ hole states, respectively, separated by the spin-orbit splitting of $1.3 \mathrm{eV}$. In the photoemission spectrum of adsorbed Xe the $p_{3 / 2}$ peak is split into two different components attributed to the lifting of the $\left|m_{j}\right|$ degeneracy due to the xenon-xenon lateral interaction $[1,2,6,7]$. As usual in photoemission of solids [8-13], in Fig. 2 the partial intensities $I_{+}$and $I_{-}$for electrons with spin parallel and antiparallel to the light helicity, respectively, are plotted by use of the total measured photoelectron intensity $I$ $=I_{+}+I_{-}$, the measured spinpolarization component $A=\left(I_{+}-I_{-}\right) /\left(I_{+}+I_{-}\right)$and the relation $I_{+,-}=0.5 I(1$ $\pm A$ ). Figure 2 shows that the $p_{1 / 2}$ peak is completely polarized parallel to the light helicity for $\Theta=0^{\circ}$.

For the off-normal studies we have only looked to the $p_{1 / 2}$ peak, because the splitting of the $p_{3 / 2}$ peak may vanish for angles different from $\Theta=0^{\circ}$ with the consequence that the polarizations of opposite sign of both $p_{3 / 2}$ peaks would partly cancel one another. Figure 2 shows that for the $p_{1 / 2}$ peak $I_{+}$continuously decreases and $I_{-}$increases with increasing $\Theta$. At about $30^{\circ}$ they are equal and at higher angles $I_{-}$dominates. Taking into account that this $p_{1 / 2}$ peak cannot be split in peaks of opposite spin directions, these results demonstrate that the spin polarization has to be given by an angle-

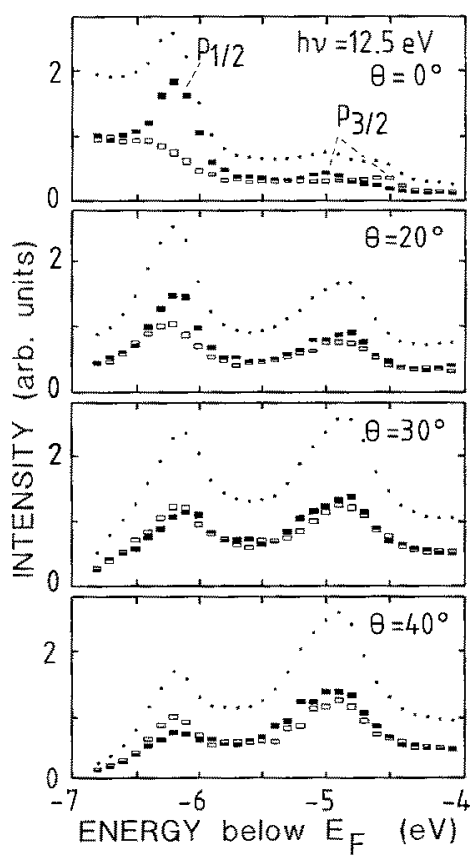

Fig. 2. Photoemission spectra at various polar emission angles $\Theta$ and photon energy $12.5 \mathrm{eV}$ for normal incidence of circularly polar. ized light. The photoemission plane is a mirror plane of the $\mathrm{Xe}(\sqrt{3 x} \sqrt{3}) R 30^{\circ} / \mathrm{Pd}(111)$ system. Filled and open squares correspond to the partial intensities $I_{+}$and $I_{-}$, totally polarized parallel and antiparallel to the helicity of light, respectively. Points are the total intensity. The height of the symbols represents the single statistical error of the counting rates. The energy scale refers to the Fermi level of the substrate. The intensity scales are arbitrary 
dependent mixture of quantities concerning energy degenerated final states.

This behavior is well known in the spin dependent photoionization of free atoms (e.g. Xe $[24,25]$ ), where the components of the spin polarization vector are functions of the emission angle $\Theta$ :

$A(\Theta)=\frac{A-\alpha\left(\frac{3}{2} \cos ^{2} \Theta-\frac{1}{2}\right)}{1-\frac{\beta}{2}\left(\frac{3}{2} \cos ^{2} \Theta-\frac{1}{2}\right)}$

component parallel

to the light helicity

$$
P_{p}(\Theta)=\frac{-\frac{3}{2} \alpha \sin \Theta \cos \Theta}{1-\frac{\beta}{2}\left(\frac{3}{2} \cos ^{2} \Theta-\frac{1}{2}\right)}
$$

component perpendicular

to the light direction

but in reaction plane

$$
P_{\perp}(\Theta)=\frac{2 \xi \sin \Theta \cos \Theta}{1-\frac{\beta}{2}\left(\frac{3}{2} \cos ^{2} \Theta-\frac{1}{2}\right)}
$$

component normal to the reaction plane

The dynamical photoionization parameters $A, \alpha, \beta, \xi$ are functions of the matrix elements $M_{s}$ and $M_{d}$ for dipole transitions from the bound $p$ state to the $s$ and $d$ continuum, respectively, and the corresponding phase shift difference $\Delta_{s d}$ between the $s$ and $d$ continuum wavefunctions.

We take the adsorbate data of Fig. 2 and, after subtraction of the inelastic background, compare it with the spin resolved photoionization data of free Xe-atoms $[24,25]$. This cross comparison shown in Fig. 3 for the spin polarization component $A(\Theta)$ shows agreement with respect to the shape of the angular dependence. $A(\Theta)$ being +1 at $\Theta=0^{\circ}$ for all energies drops down to the negative value -0.5 with increasing $\Theta$. The angle, where $A(\Theta)$ vanishes, increases with decreasing photon energy, in the gas phase $[24,25]$ as well as in the adsorbate phase. Adsorbate data for the photon energies from 12 to $21 \mathrm{eV}$ are shown in Fig. 3. The photoemission data of the Xe adsorbate can be fitted by use of the formula given above for the gas phase. Fig. 3 additionally shows the results of these fit procedures as full curves. There exists some agreement between gas phase results at photon energy $15.5 \mathrm{eV}$ and adsorbate results at $12.5 \mathrm{eV}$. This different photon energy results in similar kinetic energies of the photoelectrons.

Also the spin polarization component $P_{\perp}$ perpendicular to the reaction plane exists in the photoemission of the adsorbate. This component, which is independent of the helicity of the radiation, is forbidden in the photoexcitation of an infinite three dimensional centro-symmetric solid $[14,16]$. Figure 4 shows the comparison
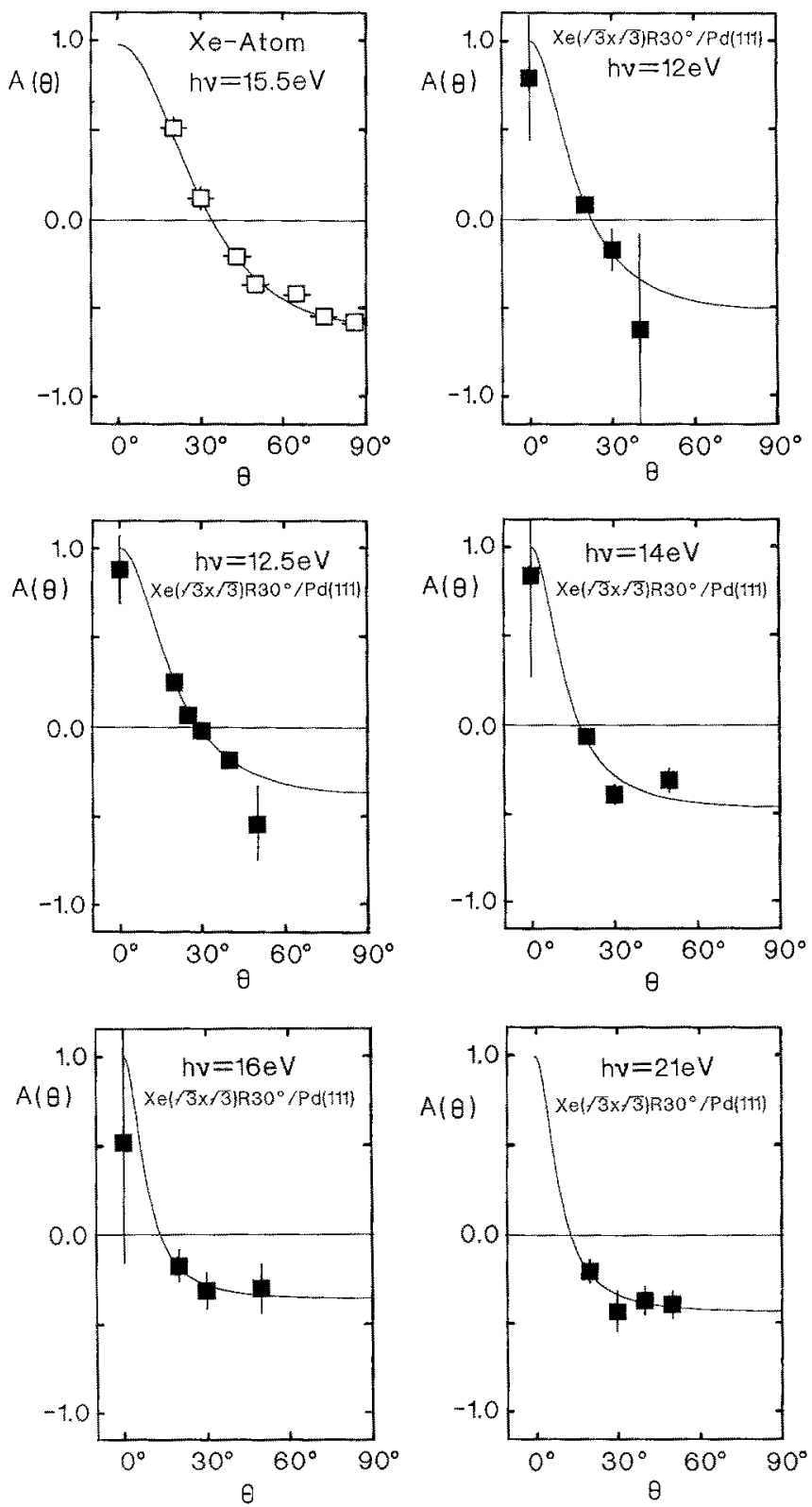

Fig. 3. Spin polarization component $A(\Theta)$ (parallel to the light helicity) as a function of the polar angle $\Theta$ : uppermost left: photoionization of Xe atoms, final ionic state $\mathrm{Xe}^{+2} P_{1 / 2}$, photon energy $15.5 \mathrm{eV}[24,25]$; other: photoemission from adsorbed $\mathrm{Xe}\left(p_{1 / 2}\right)$ at different photon energies. The size of the symbols corresponds to the experimental uncertainties

of $P_{\perp}\left(\Theta=30^{\circ}\right)$ as function of the light energy between gas phase and adsorbate. In the gas phase $P_{\perp}$ (described by $\xi$ ) is a direct measure for the interference term between the $s$ and $d$ partial waves; it is proportional to $M_{s} \cdot M_{d} \cdot \sin A_{s d}$. The quantitative agreement between the data for free and adsorbed atom in Fig. 4 indicates that this might be true also for our adsorbate system.

Figure 5 shows in details the angular dependence of $P_{\perp}(\Theta)$ for different photon energies in photoemission of the Xenon adsorbate. While for $h v=12.5 \mathrm{eV}$ this almost vanishes the experimental result at $h v=14 \mathrm{eV}$ is in some agreement with the gas phase data (at $h v=15.5 \mathrm{eV}$ ) also 


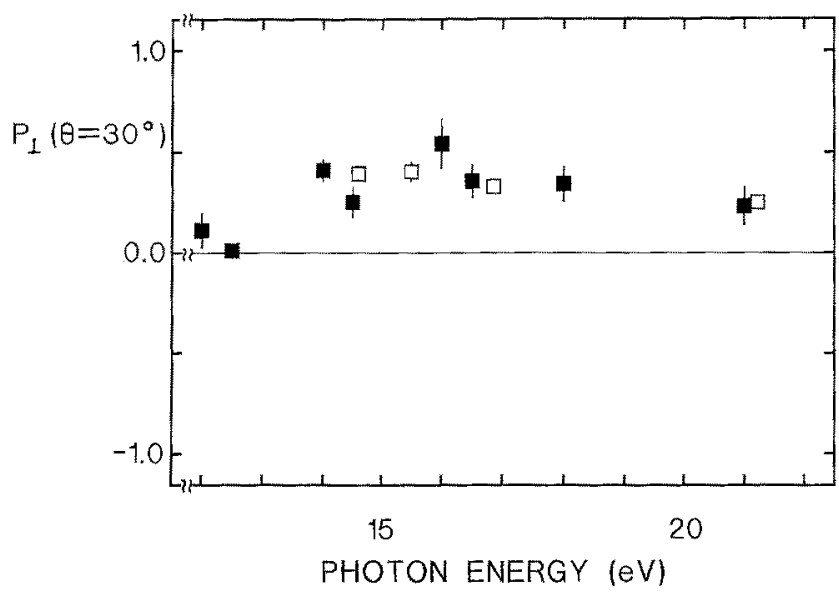

Fig. 4. Polarization component $P_{\perp}$ (perpendicular to the reaction plane) for photoemission of $\mathrm{Xe}\left(p_{1 / 2}\right)$ as function of the photon energy at a polar emission angle of $\Theta=30^{\circ}$. Filled squares show the experimental data for the adsorbate system. Open squares show the corresponding $P_{\perp}$ results for free Xe-atoms $[28,29]$. The size of the symbols corresponds to the experimental uncertainties

given in Fig. 5 (above left). All the curves in Fig. 5 are fits based upon the value at $\Theta=30^{\circ}$ and the formula given above for the gas phase which includes $P_{\perp}(\Theta=0)$ $=0$. While the comparison between the experimental data and these fit curves works quite well for photon energies below $14 \mathrm{eV}$ there are some discrepancies in the shape of the angular dependence at higher energies.

\section{Determination of the dynamical parameters and the corresponding amplitudes and phase shifts for the adsorbate photoemission}

As the fit procedure of the adsorbate data did roughly work according to the formulas describing the gas phase photoionization, the "dynamical photoemission parameters" $A, \alpha, \beta, \xi$ can be extracted. In Fig. 6 they are shown as full points and are compared at various photon energies with the gas phase data. While $A$, which does not depend upon the phase shift difference $\boldsymbol{A}_{s d}$, demonstrates agreement between free and adsorbed atoms, the parameters $\alpha$ and $\beta$, which depend upon $\Delta_{s d}$, show some discrepancies. As stated above for $P_{\perp}, \xi$ also depends on $\Delta_{s d}$. The better agreement for $\xi$ between adsorbate- and free atom data than for $\alpha$ and $\beta$ may be due to the fact that $\xi$ is related to $\Delta_{s d}$ via $\sin \Delta_{s d}$ while $\alpha$ and $\beta$ are related to $\Delta_{s d}$ via $\cos \Delta_{s d}[24,25]$.

From the data for the dynamical parameters $A$ and $\xi$ the ratio of the squared dipole matrix elements $\left(Q_{d} / Q_{s}=D_{4}^{2} / D_{5}^{2}\right)[24,25]$ for the transitions of the bound $p$-state into the energy degenerate $d$ - and s-continua and their relative phase shift difference $\left(\Delta_{d s}=\left(\delta_{4}-\delta_{5}\right)\right)$ can be extracted. They are given in Fig. 7 as full points with the corresponding error bars and are shown together with the corresponding gas phase data (open symbols). This cross comparison between adsorbate and gas phase data shows reasonable agreement. In Fig. $7 \mathrm{~b}$ the full and dash-dotted curves are lines to guide the eyes for the energy dependence of the phase shift difference of adsorbate and gas phase photoelectron emission, respectively.
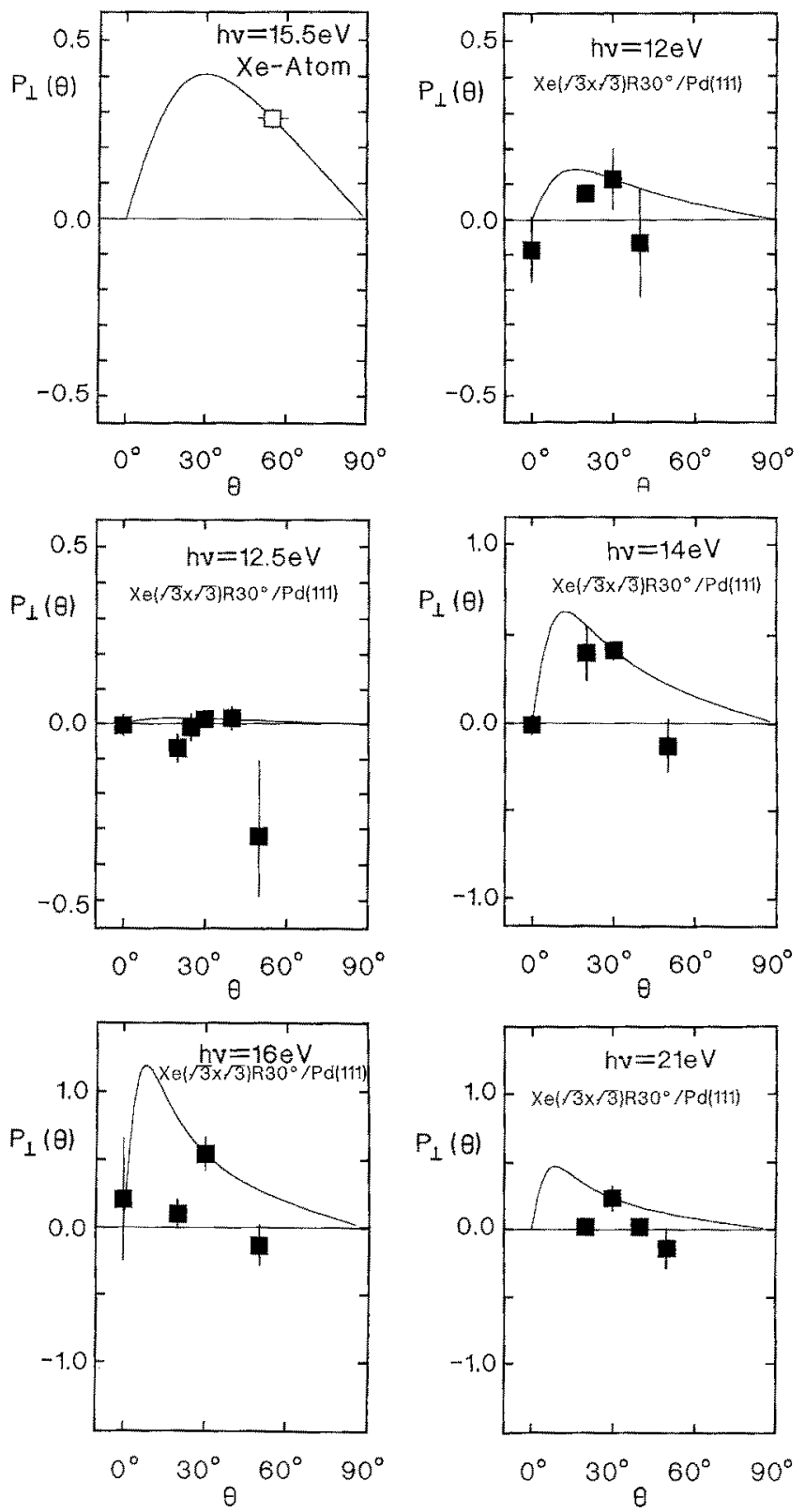

Fig. 5. As Fig. 3 but the spin polarization component perpendicular to the reaction plane. The uppermost left part shows the gas phase data $[24,25]$; the other the adsorbate photoemission

The dotted and the dashed curves show the energy dependence of the Coulomb phase shift difference only (right scale) for the photoionization thresholds 13.44 and $11.4 \mathrm{eV}$ for gas phase and adsorbate photoemission, respectively. This demonstrates, that the Coulomb phase shift difference is the largest part of the phase-shift differences between $d$ and $s$ continuum. After subtraction of the Coulomb phase shift difference one obtains the difference of the quantum defects as given in Fig. $7 \mathrm{c}$. Even there the agreement between the adsorbate data and the gas phase results is satisfactory.

Because the same matrix elements squares $Q_{d}$ and $Q_{s}$ as well as the same phase shift difference $\Delta_{d s}$ describes $A$ and $\xi$ as well as $\alpha$ and $\beta[24,25]$ values for $\alpha$ and $\beta$ can also be calculated from the given data in Fig. 7 a and Fig. $7 \mathrm{~b}$. These calculated values and their uncertain- 

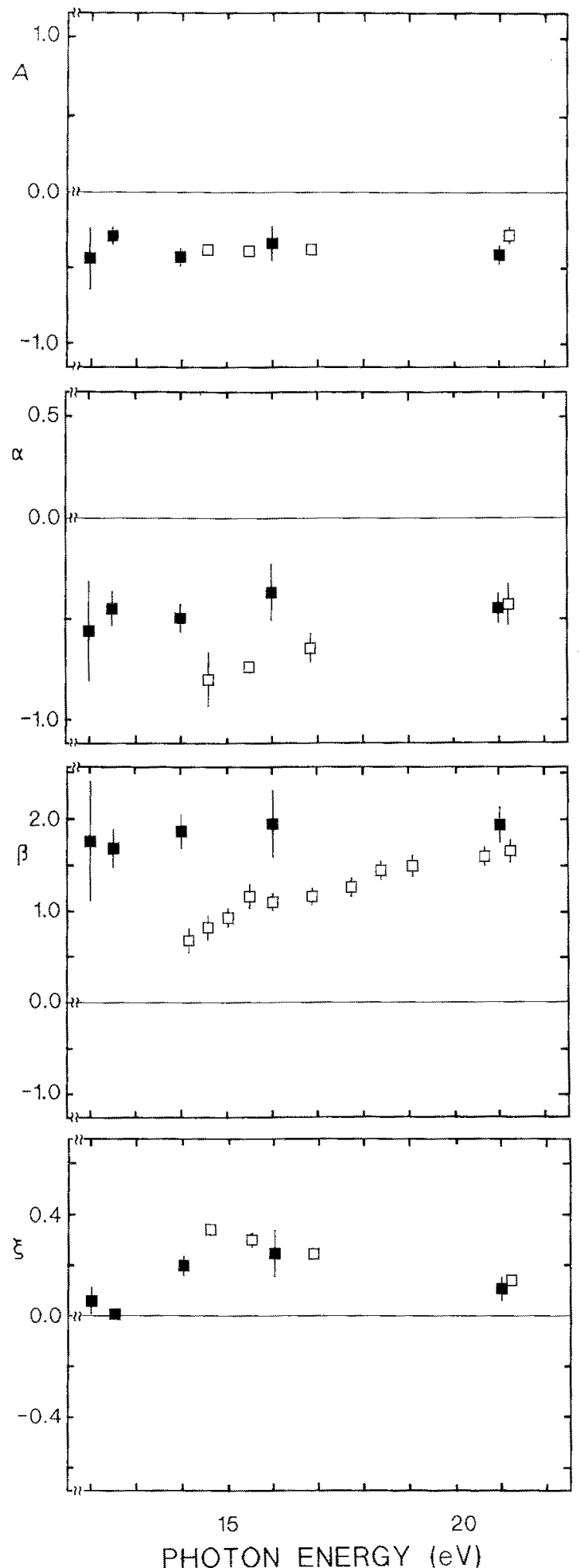

Fig. 6. Spin polarization parameters $A, \alpha, \beta$ and $\xi$ as a function of photon energy. Filled squares result from fit procedures of the adsorbate photoemission data. The heigth of the symbols corresponds to the standard deviation of the fit procedures. Open squares show the parameters in the free atom case $(A, \alpha:[24,25]$, $\beta:[30], \xi:[28,29]$

ties are shown in Fig. 8 as open symbols; they are compared with the directly determined values (full points) from Fig. 6 (middle parts, obtained from the direct measured quantities) and show reasonable agreement. a)

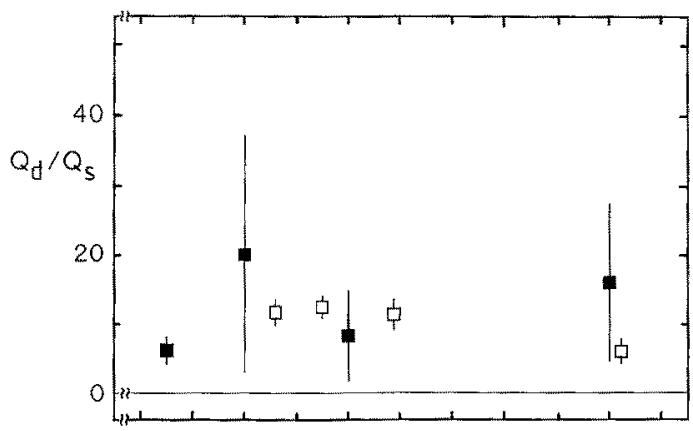

b)

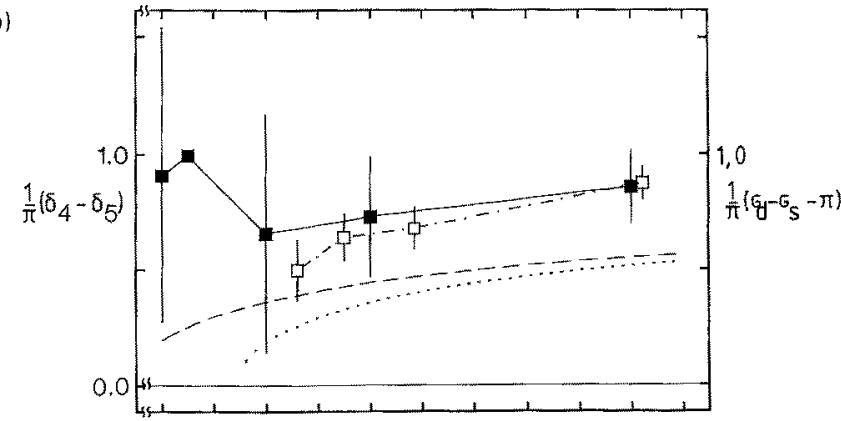

c)

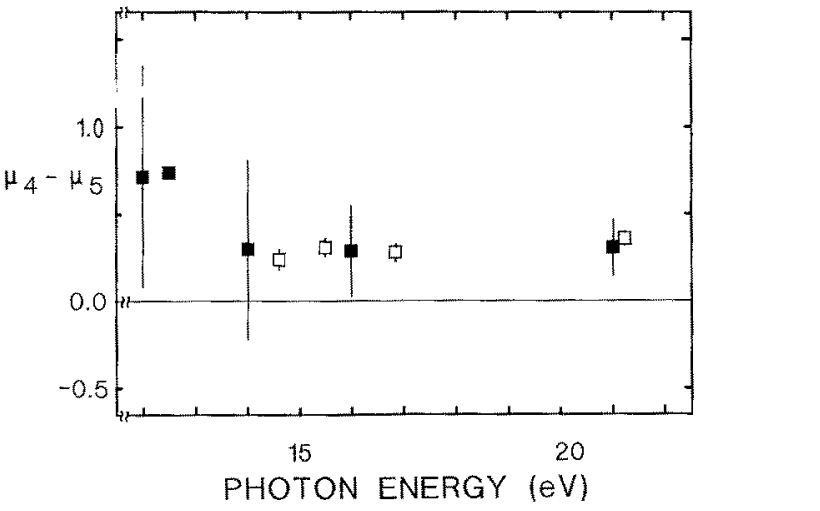

Fig. 7. a) ratio of squares of matrix elements $Q_{d} / Q_{s}$ obtained from $A$ in Fig. 6 ; b) Phase shift differences $1 / \pi\left(\delta_{4}-\delta_{5}\right)=1 / \pi\left(\delta_{d}-\delta_{s}\right)$ obtained from $A$ and $\xi$ in Fig. 6; curves see text; c) difference of quantum defects (phases after subtraction of Coulomb phase shifts)

Last but not least it is worth noting, that the agreement between gas phase and adsorbate in the angular dependence of photoemission is only good for the spin polarization components and their corresponding dynamical parameters; it does not hold for the intensity distribution. Figure 9 shows the dependence of the photoelectron intensity upon the emission angle for the $\mathrm{Xe} \sqrt{3 x} \sqrt{3} \mathrm{R} 30^{\circ}$ adsorbate on $\mathrm{Pd}(111)$ in a polar plot for the two photon energies $h v=12 \mathrm{eV}$ and $21 \mathrm{eV}$. For $h v=21 \mathrm{eV}$ the photoemission intensity vanishes for $\Theta=0^{\circ}$ as well for $\Theta=90^{\circ}$ which cannot be explained by the well known gas phase intensity distribution

$I(\Theta) \propto 1-\beta / 2\left(3 / 2 \cos ^{2} \Theta-1 / 2\right)$.

Note that the intensity distribution $I(\Theta)$ for adsorbates may contain an additional factor of $\sin \theta \cos \theta$ due to the existence of the crystal surface which seems to be spin independent because the spin polarization components of the adsorbate photoelectron angular resolved emissions fit well to the gas phase data as shown above. 

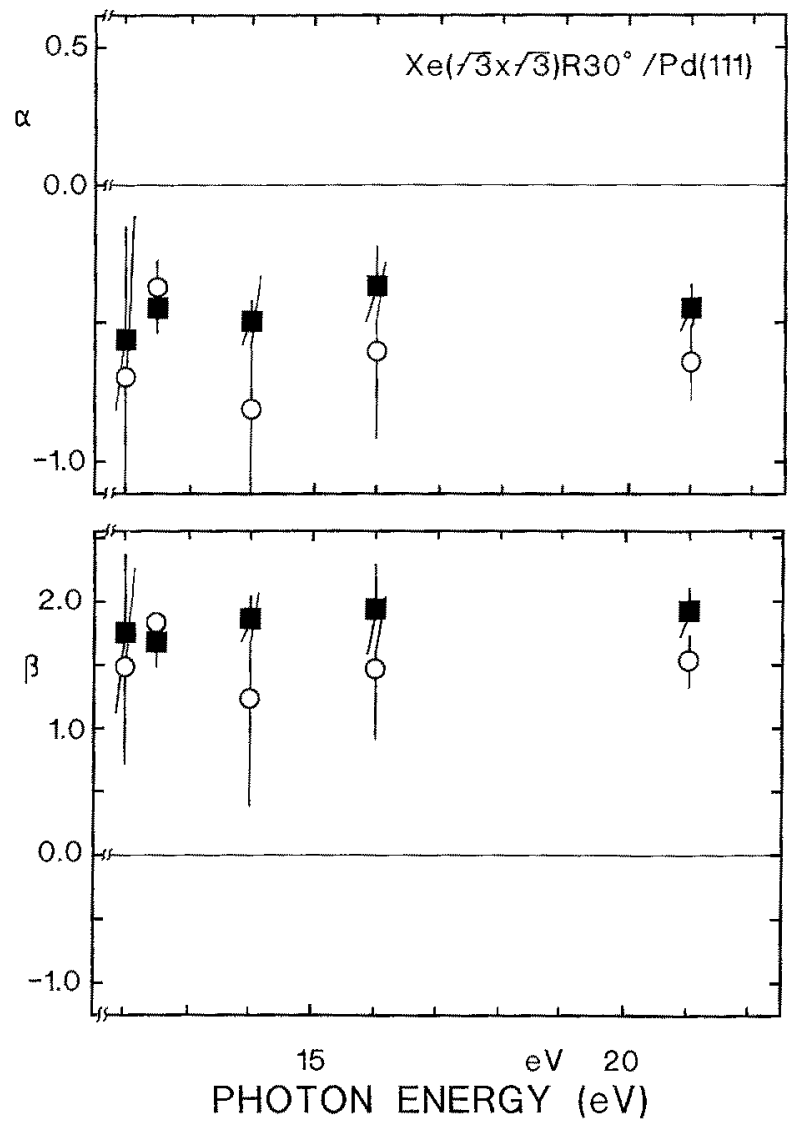

Fig. 8. Comparison of the dynamical parameters $\alpha$ and $\beta$ for $p_{1 / 2}$ photoemission of adsorbates obtained from the data of Fig. 7 (open points) with those directly measured (given in Fig. 6)

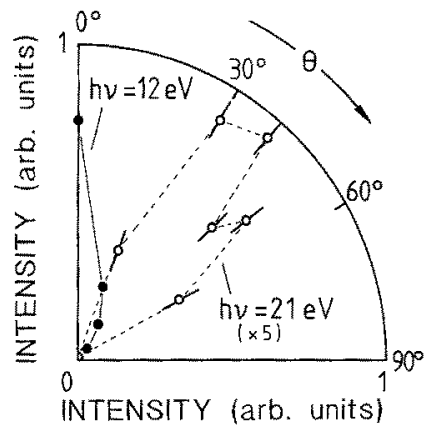

Fig. 9. Polar plot of the photoelectron intensity of xenon $p_{1 / 2}$ adsorbates photoemission (after inelastic background subtraction) as function of the emission angle $\Theta$. The lines connect the experimental data in order to guide the eyes; the values for $h v=21 \mathrm{eV}$ are enlarged by a factor of 5 compared with those for $h y=12 \mathrm{eV}$

\section{Summary}

The angular dependences of the spin polarization components of photoelectrons from Xenon adsorbates show agreement with the corresponding data of gas phase photoionizaton. The curves can be well fitted with the corresponding angular dependence curves from the isolated atom ionization yielding the so called "dynamical parameters" of adsorbate photoionization which also agree with the gas phase data. While this cross comparison well works for the spin polarization values it fails for the non-spin resolved intensity, which again shows, that opposite to the intensity the spin polarization of photoelectrons based upon the spin orbit interaction may be rather an atomic effect than given by the long distance order of the crystal surface. This holds especially for the case the adsorbate is very weakly bound to the crystal surface and thus not strongly influenced in its electronic structure as in the case of van der Waals adsorbed rare gases.

This work was supported by BMFT (05431). We thank the BESSY staff for successful cooperation.

\section{References}

1. Schönhense, G., Eyers, A., Friess, U., Schäfers, F., Heinzmann, U.: Phys. Rev. Lett. 54, 547 (1985)

2. Schönhense, G., Eyers, A., Heinzmann, U.: Phys. Rev. Lett. 56, $512(1986)$

3. Schmiedeskamp, B., Kessler, B., Vogt, B., Heinzmann, U.: Surf. Sci. 223, 465 (1989)

4. Feder. R.: Sol. State. Comm. 28, 27 (1978)

5. Heinzmann, U., Schönhense, G.: In: Polarized electrons in surface physics. Feder, R. (ed.) p. 467. Singapore: World Scientific 1985

6. Schönhense, G.: Appl. Phys. A 41, 39 (1986)

7. Heinzmann, U.: Phys. Scr. T17, 77 (1987)

8. Eyers, A., Schäfers, F., Schönhense, G., Heinzmann, U., Oepen, H.P., Hünlich, K., Kirschner, J., Borstel, G.: Phys. Rev, Lett. 52, 1559 (1984)

9. Müller, N., Kessler, B., Schmiedeskamp, B., Schönhense, G., Heinzmann, U.: Sol. State Comm. 61, 187 (1987)

10. Kessler, B., Eyers, A., Horn, K., Müller, N., Schmiedeskamp, B., Schönhense, G., Heinzmann, U.: Phys. Rev. Lett. 59, 331 (1987)

11. Tamura, E., Feder, R., Vogt, B., Schmiedeskamp, B., Heinzmann, U.: Z. Phys. B - Condensed Matter 77, 129 (1989)

12. Garbe, J., Kirschner, J.: Phys. Rev. B39, 1530 (1989)

13. Garbe, J., Kirschner, J.: Phys. Rev. B39, 6115 (1989)

14. Feder, R.: Polarized electrons in surface physics. Singapore: World Scientific 1985 and references therein

15. Kirschner, J.: Polarized electrons at surfaces. Springer tracts in modern physics, Vol. 106. Berlin, Heidelberg, New York: Springer 1985

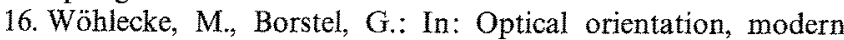
problems in condensed matter sciences. Meier, F., Zakharchenya, B.P. (eds.), p. 423. Amsterdam; Elsevier 1984

17. Feder, R., Gudat, W., Kisker, E., Rodrigues, A., Schröder, K. Sol. State Comm. 46, 619 (1983) and references therein

18. Oepen, H.P., Hünlich, K., Kirschner, J.: Phys. Rev. Lett. 56, $496(1986)$

19. Oepen, H.P., Hünlich, K., Kirschner, J., Eyers, A., Schäfers, F.: Sol, State Comm. 59, 521 (1986)

20. Garbe, J., Kirschner, J.: Phys. Rev. B39, 9859 (1989)

21. Feder, R.: Sol. State Comm. 21, 1091 (1977)

22. Kessler, J.: Polarized electrons 2nd Edn. Berlin, Heidelberg, New York: Springer 1985

23. Ignatjevs, A., Pendry, J.B., Rhodin, T.N.: Phys. Rev. Lett. 26, 189 (1971)

24. Heckenkamp, Ch., Schäfers, F., Schönhense, G., Heinzmann, U.: Phys. Rev. Lett. 52, 421 (1984)

25. Heckenkamp, Ch., Schäfers, F., Schönhense, G., Heinzmann, U.: Z. Phys. D - Atoms, Molecules and Clusters 2, 257 (1986)

26. Schäfers, F., Peatman, W., Eyers, A., Heckenkamp, Ch., Schönhense, G., Heinzmann, U.: Rev. Sci. Instrum. 57, 1032 (1986)

27. Jost, K.: J. Phys. E12, 1001, 1006 (1976)

28. Heinzmann, U., Schönhense, G., Kessler, J.: Phys. Rev, Lett. 42, $1603(1979)$

29. Schönhense, G.: Phys. Rev. Lett. 44, 640 (1980)

30. Holland, D.M.P., Parr, A.C., Ederer, D.L., Dehmer, J.L., West, J.B.: Nucl, Instrum. Methods 195, 331 (1982) 